\title{
A NOTE ON CONVEX FUNCTIONS
}

\author{
YU-RU SYAU
}

(Received 30 June 1997 and in revised form 15 June 1998)

\begin{abstract}
In this paper, we give two weak conditions for a lower semi-continuous function on the $n$-dimensional Euclidean space $R^{n}$ to be a convex function. We also present some results for convex functions, strictly convex functions, and quasi-convex functions.
\end{abstract}

Keywords and phrases. Convex functions, lower semi-continuous functions, quasi-convex functions.

1991 Mathematics Subject Classification. 26B25, 52A41.

1. Introduction. Let $E$ be a normed vector space over the real number system $R^{1}$. An extended-real-valued function $f: E \rightarrow[-\infty,+\infty]$ is said to be convex if for all $x, y \in E$ and all $\alpha, u, v \in R^{1}$ such that $f(x)<u, f(y)<v, 0<\alpha<1$,

$$
f(\alpha x+(1-\alpha) y)<\alpha u+(1-\alpha) v .
$$

Let us recall the definitions of strictly convex functions and quasi-convex functions.

DEFINITION 1.1. Let $f$ be a function from $E$ to $R^{1}$.

(1) $f$ is said to be strictly convex if

$$
f(\alpha x+(1-\alpha) y)<\alpha f(x)+(1-\alpha) f(y)
$$

for every $x \in E, y \in E, x \neq y$, and $\alpha \in(0,1)$.

(2) $f$ is said to be quasi-convex if

$$
f(\alpha x+(1-\alpha) y) \leq \max \{f(x), f(y)\}
$$

for any $x, y \in E$ and $\alpha \in[0,1]$; and strongly quasi-convex if strict inequality holds for all $x, y \in E, x \neq y$ and $\alpha \in(0,1)$.

(3) $f$ is said to be strictly quasi-convex if

$$
f(\alpha x+(1-\alpha) y)<\max \{f(x), f(y)\}
$$

for all $x, y \in E, f(x) \neq f(y)$, and $\alpha \in(0,1)$.

Recall that, by definition, an extended-real-valued function $f$ defined on a set $S \subset E$ is to be lower semi-continuous at a point $x \in S$ if, for each $\lambda \in R^{1}, \lambda<f(x)$, there exists a neighborhood $U$ of $x$ such that $f(y)>\lambda$ for all $y \in U . f: S \rightarrow[-\infty,+\infty]$ is said to be lower semi-continuous if $f$ is lower semi-continuous at each point of $S$.

This paper is organized as follows. In Section 2, we review some basic results for convex functions and lower semi-continuous functions. Section 3 gives two weak conditions for a lower semi-continuous function to be a convex function and presents a 
result which provides an important connection between convex functions and strictly convex functions. Some properties of quasi-convex functions, strongly quasi-convex functions, and strictly quasi-convex functions are investigated in Section 4 . The concept of convexity is important for quantitative and qualitative studies in mathematical programming involving convex functions. Our results are motivated by Yang $[4,5]$.

2. Preliminaries. First, we recall the following two properties which characterize lower semi-continuous functions (see Tiel [3]).

THEOREM 2.1. Let $S \subset E$. A function $f: S \rightarrow[-\infty,+\infty]$ is lower semi-continuous if and only if, for every real number $\lambda$, the set $\{x \in S: f(x) \leq \lambda\}$ is closed.

THEOREM 2.2. Let $S \subset E$. A function $f: S \rightarrow[-\infty,+\infty]$ is lower semi-continuous if and only if its epigraph epi $(f)=\left\{(x, \lambda) \in S \times R^{1}: f(x) \leq \lambda\right\}$ is closed (as a subset of $\left.S \times R^{1}\right)$.

We recall the following:

THEOREM 2.3. A function $f: E \rightarrow[-\infty,+\infty]$ is convex if and only if its epigraph $\operatorname{epi}(f)=\left\{(x, \lambda): x \in E, \lambda \in R^{1}, f(x) \leq \lambda\right\}$ is convex as a subset of $E \times R^{1}$.

THEOREM 2.4. A function $f: E \rightarrow(-\infty,+\infty]$ is convex if and only if

$$
f(\alpha x+(1-\alpha) y) \leq \alpha f(x)+(1-\alpha) f(y)
$$

for all $x$ and $y \in E$ and all $\alpha \in(0,1)$.

It follows from Theorem 2.3 that $f$ is convex if its epigraph is a singleton or an empty set.

Definition 2.1. Let $(x, s),(y, t) \in R^{n+1}$, with $x, y \in R^{n}$ and $s, t \in R^{1}$. The line segment $[(x, s),(y, t)]$ (with endpoints $(x, s)$ and $(y, t))$ is the segment $\{\gamma(x, s)+$ $(1-\gamma)(y, t): 0 \leq \gamma \leq 1\}$. If $(x, s) \neq(y, t)$, the interior $((x, s),(y, t))$ of $[(x, s),(y, t)]$ is the segment $\{\gamma(x, s)+(1-\gamma)(y, t): 0<\gamma<1\}$. In a similar way, we can define $[(x, s),(y, t))$ and $((x, s),(y, t)]$.

3. Convex functions. This section gives some properties on convex functions and strictly convex functions. Our results greatly simplify the criteria on convex functions and strictly convex functions.

By using Theorems 2.2 and 2.3, one can prove the following two results which give weak conditions for a lower semi-continuous function to be a convex function.

THEOREM 3.1. Let $f: R^{n} \rightarrow[-\infty,+\infty]$ be lower semi-continuous and suppose that there exists an $\alpha \in(0,1)$ such that for all $x, y \in R^{n}, u, v \in R^{1}$ such that $f(x)<u$, $f(y)<v$,

$$
f(\alpha x+(1-\alpha) y)<\alpha u+(1-\alpha) v .
$$

Then $f: R^{n} \rightarrow[-\infty,+\infty]$ is convex.

Proof. By Theorem 2.3, it is sufficient to show that epi $(f)$ is convex as a subset of $R^{n+1}$. By contradiction, suppose that there exist $\left(x, \lambda_{1}\right),\left(y, \lambda_{2}\right) \in \operatorname{epi}(f)$ (with $x, y \in$ 
$R^{n}$ and $\left.\lambda_{1}, \lambda_{2} \in R^{1}\right)$ and $\alpha_{0} \in(0,1)$ such that

$$
\alpha_{0}\left(x, \lambda_{1}\right)+\left(1-\alpha_{0}\right)\left(y, \lambda_{2}\right) \notin \operatorname{epi}(f) .
$$

Let $x_{0}=\alpha_{0} x+\left(1-\alpha_{0}\right) y$ and $\lambda_{0}=\alpha_{0} \lambda_{1}+\left(1-\alpha_{0}\right) \lambda_{2}$, then $\left(x_{0}, \lambda_{0}\right) \notin$ epi $(f)$. Let

$$
A=\operatorname{epi}(f) \cap\left[\left(x, \lambda_{1}\right),\left(x_{0}, \lambda_{0}\right)\right]
$$

and

$$
B=\operatorname{epi}(f) \cap\left[\left(x_{0}, \lambda_{0}\right),\left(y, \lambda_{2}\right)\right] .
$$

Since $f: R^{n} \rightarrow[-\infty,+\infty]$ is lower semi-continuous, by Theorem 2.2, epi $(f)$ is a closed subset of $R^{n+1}$. Consequently, $A$ and $B$ are bounded and closed subsets of $R^{n+1}$, and $\left(x_{0}, \lambda_{0}\right) \notin A,\left(x_{0}, \lambda_{0}\right) \notin B$. Thus, there exist $(\tilde{x}, s) \in A$ and $(\tilde{y}, t) \in B$, with $\tilde{x}, \tilde{y} \in R^{n}$ and $s, t \in R^{1}$, such that

$$
\min _{a \in A}\left\|a-\left(x_{0}, \lambda_{0}\right)\right\|=\left\|(\tilde{x}, s)-\left(x_{0}, \lambda_{0}\right)\right\|
$$

and

$$
\min _{b \in B}\left\|b-\left(x_{0}, \lambda_{0}\right)\right\|=\left\|(\tilde{y}, t)-\left(x_{0}, \lambda_{0}\right)\right\|,
$$

where $\|\cdot\|$ is the norm on $R^{n+1}$. Hence, we have

$$
\operatorname{epi}(f) \cap\left((\tilde{x}, s),\left(x_{0}, \lambda_{0}\right)\right]=\varnothing, \quad \operatorname{epi}(f) \cap\left[\left(x_{0}, \lambda_{0}\right),(\tilde{y}, t)\right)=\varnothing .
$$

Therefore,

$$
\operatorname{epi}(f) \cap((\tilde{x}, s),(\tilde{y}, t))=\varnothing .
$$

Noticing that $\tilde{x} \neq \tilde{y}$ and $s \neq t$, and $((\tilde{x}, s),(\tilde{y}, t)) \neq \varnothing$.

On the other hand, since $(\tilde{x}, s),(\tilde{y}, t) \in \operatorname{epi}(f)$, we have $f(\tilde{x})<s+\epsilon, f(\tilde{y})<t+\epsilon$ for each $\epsilon>0$. Since $\alpha(s+\epsilon)+(1-\alpha)(t+\epsilon)=\alpha s+(1-\alpha) t+\epsilon$, by the hypothesis of the theorem, we have

$$
f(\alpha \tilde{x}+(1-\alpha) \tilde{y})<\alpha s+(1-\alpha) t+\epsilon .
$$

Since $\epsilon$ is an arbitrary positive real number, it follows that

$$
f(\alpha \tilde{x}+(1-\alpha) \tilde{y}) \leq \alpha s+(1-\alpha) t .
$$

Hence,

$$
\alpha(\tilde{x}, s)+(1-\alpha)(\tilde{y}, t) \in \operatorname{epi}(f)
$$

which contradicts (3.8). Thus, we conclude that epi $(f)$ is convex. This completes the proof.

THEOREM 3.2. Let $f: R^{n} \rightarrow(-\infty,+\infty]$ be lower semi-continuous. Then $f$ is convex if and only if, for all $x_{1}, x_{2} \in R^{n}$, there exists an $\alpha \in(0,1)$ ( $\alpha$ depends on $x_{1}, x_{2}$ ) such that

$$
f\left(\alpha x_{1}+(1-\alpha) x_{2}\right) \leq \alpha f\left(x_{1}\right)+(1-\alpha) f\left(x_{2}\right) .
$$

Proof. Let $f: R^{n} \rightarrow(-\infty,+\infty]$ be convex. From Theorem 2.4, it follows that, for all $x_{1}, x_{2} \in R^{n}$, there exists an $\alpha \in(0,1)$ such that (3.12) holds. 
For the converse part, by Theorem 2.3, it is sufficient to show that epi $(f)$ is convex as a subset of $R^{n+1}$. By contradiction, suppose that there exist $\left(x, \lambda_{1}\right),\left(y, \lambda_{2}\right) \in \operatorname{epi}(f)$ (with $x, y \in R^{n}$ and $\lambda_{1}, \lambda_{2} \in R^{1}$ ), and $\alpha_{0} \in(0,1)$ such that

$$
\alpha_{0}\left(x, \lambda_{1}\right)+\left(1-\alpha_{0}\right)\left(y, \lambda_{2}\right) \notin \operatorname{epi}(f) .
$$

Let $x_{0}=\alpha_{0} x+\left(1-\alpha_{0}\right) y$ and $\lambda_{0}=\alpha_{0} \lambda_{1}+\left(1-\alpha_{0}\right) \lambda_{2}$, then $\left(x_{0}, \lambda_{0}\right) \notin$ epi $(f)$. We follow the proof of Theorem 3.1. Having defined $A, B,(\tilde{x}, s),(\tilde{y}, t)$, we find that

$$
\operatorname{epi}(f) \cap((\tilde{x}, s),(\tilde{y}, t))=\varnothing .
$$

Notice that $((\tilde{x}, s),(\tilde{y}, t)) \neq \varnothing$.

On the other hand, by the hypothesis of the theorem, for $\tilde{x}, \tilde{y} \in R^{n}$, there exists an $\alpha \in(0,1)$ such that

$$
f(\alpha \tilde{x}+(1-\alpha) \tilde{y}) \leq \alpha f(\tilde{x})+(1-\alpha) f(\tilde{y}) .
$$

Since $(\tilde{x}, s),(\tilde{y}, t) \in \operatorname{epi}(f)$, we have

$$
f(\tilde{x}) \leq s \quad \text { and } \quad f(\tilde{y}) \leq t .
$$

Combining (3.15) and (3.16), we obtain

$$
f(\alpha \tilde{x}+(1-\alpha) \tilde{y}) \leq \alpha s+(1-\alpha) t .
$$

So, $\alpha(\tilde{x}, s)+(1-\alpha)(\tilde{y}, t) \in \operatorname{epi}(f)$, which contradicts (3.14). Thus, we conclude that epi $(f)$ is convex. This completes the proof.

REMARK 3.1. We point out that there are functions, which are not lower semicontinuous and satisfy the conditions of Theorem 3.2 (i.e., the weak conditions that a lower semi-continuous function on $R^{n}$ is a convex function) but not convex. For example, consider the function $f: R^{1} \rightarrow R^{1}$ defined by

$$
f(x)= \begin{cases}\frac{1}{4}, & \text { if } x<0 \\ \frac{1}{2}+x, & \text { if } x \geq 0\end{cases}
$$

Proof. (a) $f$ is not lower semi-continuous since the set $\left\{x \in R^{1}: f(x) \leq 1 / 4\right\}$ is not a closed subset of $R^{1}$.

(b) To show that $f$ satisfies the conditions of Theorem 3.1, it suffices to show that, for all $x_{1}<0, x_{2} \geq 0$, there exists an $\alpha \in(0,1)$ ( $\alpha$ depends on $x_{1}, x_{2}$ ) such that

$$
f\left(\alpha x_{1}+(1-\alpha) x_{2}\right) \leq \alpha f\left(x_{1}\right)+(1-\alpha) f\left(x_{2}\right) .
$$

Let $x_{1}<0$ and $x_{2} \geq 0$, then there exists an $\alpha \in(0,1)$ ( $\alpha$ depends on $\left.x_{1}, x_{2}\right)$ such that $\alpha x_{1}+(1-\alpha) x_{2}<0$. Thus,

$$
f\left(\alpha x_{1}+(1-\alpha) x_{2}\right)=\frac{1}{4} .
$$

Moreover, since $f\left(x_{1}\right)=1 / 4$ and $f\left(x_{2}\right)=(1 / 2)+x_{2} \geq 1 / 2$,

$$
\alpha f\left(x_{1}\right)+(1-\alpha) f\left(x_{2}\right)>\frac{1}{4} \alpha+\frac{1}{4}(1-\alpha) .
$$

Combining (3.20) and (3.21), we obtain (3.19). 
(c) Finally, let us show that $f$ is not a convex function. If $x_{1}=-1 / 5, x_{2}=1 / 2$, and $\alpha=1 / 3$, then

$$
\alpha f\left(x_{1}\right)+(1-\alpha) f\left(x_{2}\right)=\frac{1}{3} \cdot \frac{1}{4}+\frac{2}{3} \cdot 1
$$

and

$$
f\left(\alpha x_{1}+(1-\alpha) x_{2}\right)=\frac{1}{2}+\frac{4}{15}=\frac{23}{30} .
$$

(3.22) and (3.23) imply that

$$
\alpha f\left(x_{1}\right)+(1-\alpha) f\left(x_{2}\right)<f\left(\alpha x_{1}+(1-\alpha) x_{2}\right),
$$

which completes the proof.

COROLlary 3.1. Let $f: R^{n} \rightarrow(-\infty,+\infty]$ be lower semi-continuous. Then $f$ is convex if and only if there exists an $\alpha \in(0,1)$ such that, for all $x_{1}, x_{2} \in R^{n}$,

$$
f\left(\alpha x_{1}+(1-\alpha) x_{2}\right) \leq \alpha f\left(x_{1}\right)+(1-\alpha) f\left(x_{2}\right) .
$$

Recall that a function $f: I \rightarrow R^{1}$, where $I$ is a (closed, open or half-open, finite or infinite) interval in $R^{1}$, is called midpoint convex if, for all $x_{1}, x_{2} \in I$,

$$
f\left(\frac{1}{2} x_{1}+\frac{1}{2} x_{2}\right) \leq \frac{1}{2} f\left(x_{1}\right)+\frac{1}{2} f\left(x_{2}\right) .
$$

It is known that if $f: I \rightarrow R^{1}$ is continuous and midpoint convex, then $f: I \rightarrow R^{1}$ is convex (see Theorem 1.10 in Tiel [3]). The following corollary generalizes this known fact.

COROLlaRY 3.2. Let $f: R^{n} \rightarrow(-\infty,+\infty]$ be lower semi-continuous. Then $f$ is convex if and only if, for all $x_{1}, x_{2} \in R^{n}$,

$$
f\left(\frac{1}{2} x_{1}+\frac{1}{2} x_{2}\right) \leq \frac{1}{2} f\left(x_{1}\right)+\frac{1}{2} f\left(x_{2}\right) .
$$

THEOREM 3.3. Let $f: R^{n} \rightarrow R^{1}$ be convex. If there exists an $\alpha \in(0,1)$ such that, for every pair of distinct points $x_{1}, x_{2} \in R^{n}$, the strict inequality

$$
f\left(\alpha x_{1}+(1-\alpha) x_{2}\right)<\alpha f\left(x_{1}\right)+(1-\alpha) f\left(x_{2}\right)
$$

holds true, then $f: R^{n} \rightarrow R^{1}$ is strictly convex.

Proof. Assume that $f: R^{n} \rightarrow R^{1}$ is a convex function which is not strictly convex. Then there exist $x, y \in R^{n}, x \neq y$, and $\gamma \in(0,1)$ such that

$$
f(\gamma x+(1-\gamma) y)=\gamma f(x)+(1-\gamma) f(y) .
$$

On the other hand, by the hypothesis of the theorem, we have

$$
f(\alpha x+(1-\alpha) y)<\alpha f(x)+(1-\alpha) f(y) .
$$

Let $z=\gamma x+(1-\gamma) y$. From equality (3.29) and inequality (3.30), it is clear that $\gamma \neq \alpha$.

(1) If $0<\gamma<\alpha$, let

$$
z_{1}=\frac{\gamma}{\alpha} x+\left(1-\frac{\gamma}{\alpha}\right) y
$$


Then $z=\gamma x+(1-\gamma) y$ can be rewritten as

$$
z=\alpha z_{1}+(1-\alpha) y
$$

According to (3.28), we have

$$
f(z)<\alpha f\left(z_{1}\right)+(1-\alpha) f(y) .
$$

Since $f$ is convex, from (3.31), we obtain

$$
f\left(z_{1}\right)<\frac{\gamma}{\alpha} f(x)+\left(1-\frac{\gamma}{\alpha}\right) f(y) .
$$

Combining (3.33) and (3.34), we obtain

$$
f(z)<\gamma f(x)+(1-\gamma) f(y)
$$

which contradicts (3.29).

(2) If $\alpha<\gamma<1$, that is

$$
0<\frac{\gamma-\alpha}{1-\alpha}<1
$$

let

$$
z_{2}=\frac{\gamma-\alpha}{1-\alpha} x+\frac{1-\gamma}{1-\alpha} y .
$$

Then $z=\gamma x+(1-\gamma) y$ can be rewritten as

$$
z=\alpha x+(1-\alpha) z_{2}
$$

According to (3.28), we have

$$
f(z)<\alpha f(x)+(1-\alpha) f\left(z_{2}\right) .
$$

Since $f$ is convex, from (3.37), we obtain

$$
f\left(z_{2}\right)<\frac{\gamma-\alpha}{1-\alpha} f(x)+\frac{1-\gamma}{1-\alpha} f(y) .
$$

Combining (3.39) and (3.40), we obtain

$$
f(z)<\gamma f(x)+(1-\gamma) f(y)
$$

which contradicts (3.29). This completes the proof.

According to Theorems 3.2 and 3.3, we have the following corollary.

COROLLARY 3.4. Let $f: R^{n} \rightarrow R^{1}$ be lower semi-continuous. If there exists an $\alpha \in$ $(0,1)$ such that, for every pair of distinct points $x_{1}, x_{2} \in R^{n}$,

$$
f\left(\alpha x_{1}+(1-\alpha) x_{2}\right)<\alpha f\left(x_{1}\right)+(1-\alpha) f\left(x_{2}\right)
$$

holds true, then $f: R^{n} \rightarrow R^{1}$ is strictly convex.

4. Quasi-convex functions. In this section, we give some properties on quasi-convex functions, strongly quasi-convex functions, and strictly quasi-convex functions. 
THEOREM 4.1. Let $f: R^{n} \rightarrow R^{1}$ be lower semi-continuous. Then $f$ is quasi-convex if and only if, for all $x_{1}, x_{2} \in R^{n}$, there exists an $\alpha \in(0,1)$ ( $\alpha$ depends on $\left.x_{1}, x_{2}\right)$ such that

$$
f\left(\alpha x_{1}+(1-\alpha) x_{2}\right) \leq \max \left\{f\left(x_{1}\right), f\left(x_{2}\right)\right\} .
$$

PRoof. It can be easily checked that $f: R^{n} \rightarrow R^{1}$ is quasi-convex if and only if, for every real number $\lambda$, the set $\left\{x \in R^{n}: f(x) \leq \lambda\right\}$ is convex. In view of this observation, it is sufficient to show that $\left\{x \in R^{n}: f(x) \leq \lambda\right\}$ is a convex set for every real number $\lambda$. By contradiction, suppose that there exists a real number $\lambda^{*}$ such that the set $F_{\lambda^{*}}=\left\{x \in R^{n}: f(x) \leq \lambda^{*}\right\}$ is not a convex set. Thus, there exist $x, y \in F_{\lambda^{*}}$, and $\alpha_{0} \in(0,1)$ such that $\alpha_{0} x+\left(1-\alpha_{0}\right) y \notin F_{\lambda^{*}}$. Let $x_{0}=\alpha_{0} x+\left(1-\alpha_{0}\right) y$, then $x_{0} \notin F_{\lambda^{*}}$. Let

$$
A=F_{\lambda^{*}} \cap\left[x, x_{0}\right] \quad \text { and } \quad B=F_{\lambda^{*}} \cap\left[x_{0}, y\right],
$$

where $\left[x, x_{0}\right]=\left\{\gamma x+(1-\gamma) x_{0}: 0 \leq \gamma \leq 1\right\}$ and $\left[x_{0}, y\right]=\left\{\gamma x_{0}+(1-\gamma) y: 0 \leq \gamma \leq 1\right\}$. Notice that $F_{\lambda^{*}}=\left\{x \in R^{n}: f(x) \leq \lambda^{*}\right\}$ is a closed set (by Theorem 2.1). Consequently, $A$ and $B$ are bounded and closed subsets of $R^{n}$, and $x_{0} \notin A, x_{0} \notin B$. Thus, there exist $\tilde{x} \in A$ and $\tilde{y} \in B$ such that

$$
\min _{a \in A}\left\|a-x_{0}\right\|=\left\|\tilde{x}-x_{0}\right\|
$$

and

$$
\min _{b \in B}\left\|b-x_{0}\right\|=\left\|\tilde{y}-x_{0}\right\|,
$$

where $\|\cdot\|$ is the norm on $R^{n}$. Hence, we have

$$
F_{\lambda *} \cap\left(\tilde{x}, X_{0}\right]=\varnothing \quad \text { and } \quad F_{\lambda *} \cap\left[X_{0}, \tilde{y}\right)=\varnothing .
$$

Therefore,

$$
F_{\lambda *} \cap(\tilde{x}, \tilde{y})=\varnothing .
$$

Notice that $\tilde{x} \neq \tilde{y}$, and so $(\tilde{x}, \tilde{y}) \neq \varnothing$.

On the other hand, by the hypothesis of the theorem, for $\tilde{x}, \tilde{y} \in R^{n}$, there exists an $\alpha \in(0,1)$ such that

$$
f(\alpha \tilde{x}+(1-\alpha) \tilde{y}) \leq \max \{f(\tilde{x}), f(\tilde{y})\} .
$$

Since $\tilde{x}, \tilde{y} \in F_{\lambda^{*}}$, we have

$$
f(\tilde{x}) \leq \lambda^{*} \quad \text { and } \quad f(\tilde{y}) \leq \lambda^{*} .
$$

Combining (4.7) and (4.8), we obtain

$$
f(\alpha \tilde{x}+(1-\alpha) \tilde{y}) \leq \lambda .
$$

So, $\alpha \tilde{x}+(1-\alpha) \tilde{y} \in F_{\lambda^{*}}$, which contradicts (4.6). Thus, we conclude that $F_{\lambda^{*}}$ is convex. This completes the proof. 
It can be easily checked that the function $f: R^{1} \rightarrow R^{1}$ defined by

$$
f(x)= \begin{cases}1, & \text { if } x=0 \\ 0, & \text { if } x \neq 0\end{cases}
$$

is strictly quasi-convex, but not quasi-convex. This shows that a strictly quasi-convex function is not necessary quasi-convex. Now, by using the technique of Yang [5, Thm. 1], we prove the following:

THEOREM 4.2. Let $f: R^{n} \rightarrow R^{1}$ be strictly quasi-convex. If there exists an $\alpha \in[0,1]$ such that, for all $x_{1}, x_{2} \in R^{n}$,

$$
f\left(\alpha x_{1}+(1-\alpha) x_{2}\right) \leq \max \left\{f\left(x_{1}\right), f\left(x_{2}\right)\right\} .
$$

Then $f: R^{n} \rightarrow R^{1}$ is quasi-convex.

Proof. By contradiction, suppose that there exist $x, y \in R^{n}$ and $\gamma \in[0,1]$ such that

$$
f(\gamma x+(1-\gamma) y)>\max \{f(x), f(y)\} .
$$

Without loss of generality, we may assume that $f(x)<f(y)$. Let $z=\gamma x+(1-\gamma) y$, then

$$
f(z)>\max \{f(x), f(y)\} .
$$

If $f(x)>f(y)$, since $f$ is strictly quasi-convex, we have $f(z)<f(x)$, which contradicts (4.13). If $f(x)=f(y)$, then (4.13) implies that

$$
f(z)>f(x)=f(y) .
$$

According to (4.11), we have

$$
f(\alpha x+(1-\alpha) y) \leq \max \{f(x), f(y)\} .
$$

From (4.14) and (4.15), it is clear that $\gamma \neq \alpha$.

(1) If $0<\gamma<\alpha$, let

$$
z_{1}=\frac{\gamma}{\alpha} x+\left(1-\frac{\gamma}{\alpha}\right) y .
$$

Then, by (4.16), $z=\gamma x+(1-\gamma) y$ can be rewritten as

$$
z=\alpha z_{1}+(1-\alpha) y
$$

According to (4.11), we have

$$
f(z) \leq \max \left\{f\left(z_{1}\right), f(y)\right\} .
$$

From (4.14) and (4.18), we obtain

$$
f(z) \leq f\left(z_{1}\right) .
$$

Let

$$
\delta=\frac{1-\alpha}{\alpha} \cdot \frac{\gamma}{1-\gamma}
$$


Since $0<\gamma<\alpha<1$, it can be easily shown that

$$
0<\delta<1 .
$$

According to $z=\gamma x+(1-\gamma) y$, (4.16), and (4.20), we have

$$
z_{1}=\delta x+(1-\delta) z
$$

Since $f$ is strictly quasi-convex, from (4.22), we obtain

$$
f\left(z_{1}\right)<\max \{f(x), f(z)\}=f(z)
$$

which contradicts (4.19).

(2) If $\alpha<\gamma<1$, that is

$$
0<\frac{\gamma-\alpha}{1-\alpha}<1
$$

let

$$
z_{2}=\frac{\gamma-\alpha}{1-\alpha} x+\frac{1-\gamma}{1-\alpha} y
$$

Thus, $z=\gamma x+(1-\gamma) y$ can be rewritten as

$$
z=\alpha x+(1-\alpha) z_{2}
$$

According to (4.11), we have

$$
f(z) \leq \max \left\{f(x), f\left(z_{2}\right)\right\} .
$$

Again, from (4.14) and (4.27), it follows that

$$
f(z) \leq f\left(z_{2}\right) .
$$

Let

$$
\eta=\frac{\gamma-\alpha}{1-\alpha} .
$$

Since $0<\alpha<\gamma<1$, it can be easily shown that $0<\eta<1$. According to $z=\gamma x+$ $(1-\gamma) y,(4.25)$, and (4.29), we have

$$
z_{2}=\eta z+(1-\eta) y .
$$

Since $f$ is strictly quasi-convex, from (4.30), we have

$$
f\left(z_{2}\right)<\max \{f(z), f(y)\}=f(z)
$$

which contradicts (4.28). This completes the proof.

Similarly, by applying the technique of Yang [5, Thms. 2,4], we have the following results:

THEOREM 4.3. Let $f: R^{n} \rightarrow R^{1}$ be quasi-convex. If there exists an $\alpha \in(0,1)$ such that, for all $x, y \in R^{n}, f(x) \neq f(y)$,

$$
f(\alpha x+(1-\alpha) y)<\max \{f(x), f(y)\}
$$

holds true, then $f: R^{n} \rightarrow R^{1}$ is strictly quasi-convex. 
THEOREM 4.4. Let $f: R^{n} \rightarrow R^{1}$ be quasi-convex. If there exists an $\alpha \in(0,1)$ such that, for every pair of distinct points $x, y \in R^{n}$,

$$
f(\alpha x+(1-\alpha) y)<\max \{f(x), f(y)\}
$$

holds true, then $f: R^{n} \rightarrow R^{1}$ is strongly quasi-convex.

\section{REFERENCES}

[1] R. T. Rockafellar, Convex Analysis, Princeton Mathematical Series, no. 28, Princeton University Press, Princeton, NJ, 1970. MR 43\#445. Zbl 193.18401.

[2] Y. R. Syau, Closed and convex fuzzy sets, Accepted by fuzzy Sets and Systems.

[3] J. van Tiel, Convex Analysis, John Wiley \& Sons, Inc., New York, 1984, An introductory text. MR 85m:49001. Zbl 565.49001.

[4] X. M. Yang, A note on convex fuzzy sets, Fuzzy Sets and Systems 53 (1993), no. 1, 117-118. MR 94b:52015. Zbl 793.04009.

[5] _ Some properties of convex fuzzy sets, Fuzzy Sets and Systems 72 (1995), no. 1, 129-132. MR 96a:52010. Zbl 851.52006.

SyAU: DePARTMENT OF INDUSTRIAL ENGINEERING, YUAN ZE UNIVERSITY, TAOYUAN, TAIWAN 320, TAIWAN 


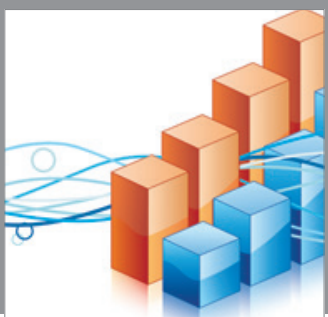

Advances in

Operations Research

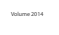

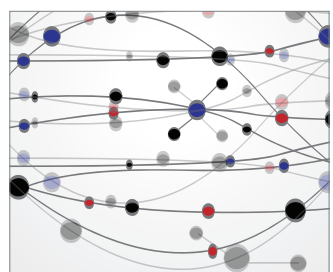

\section{The Scientific} World Journal
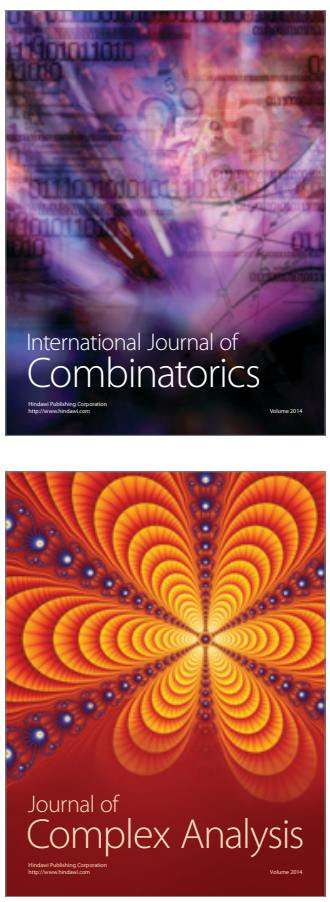

International Journal of

Mathematics and

Mathematical

Sciences
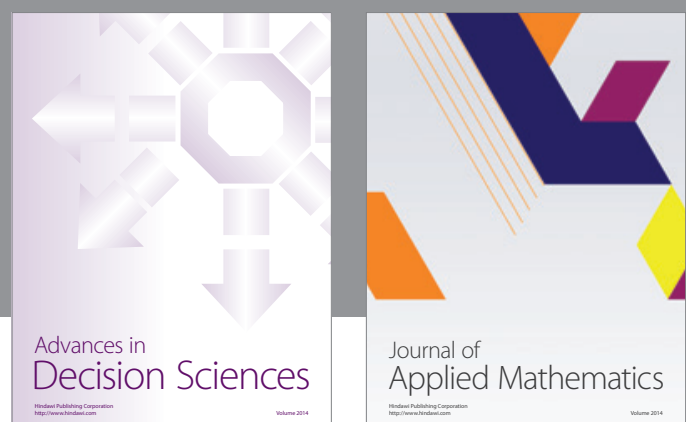

Journal of

Applied Mathematics
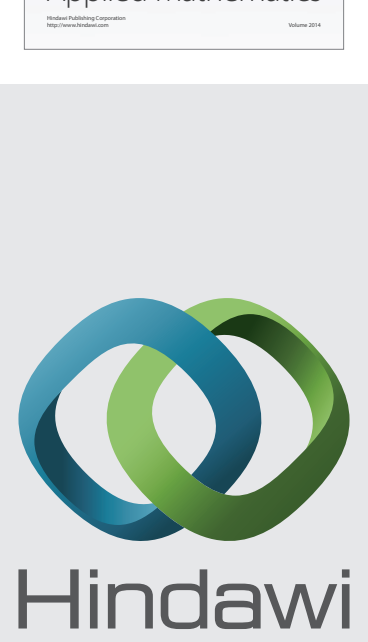

Submit your manuscripts at http://www.hindawi.com
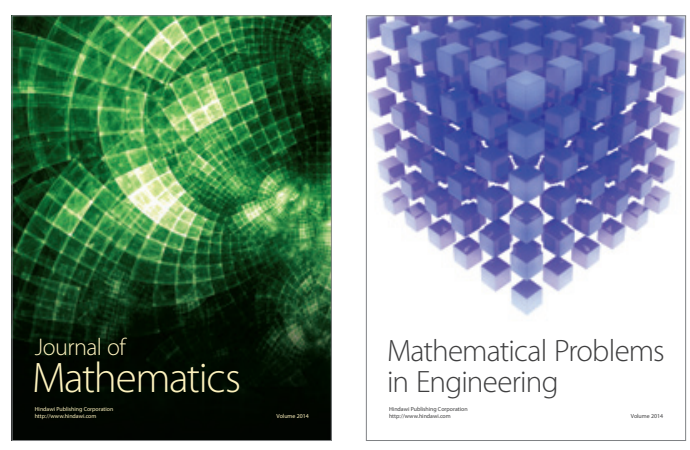

Mathematical Problems in Engineering
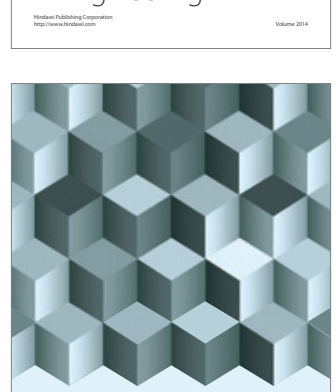

Journal of

Function Spaces
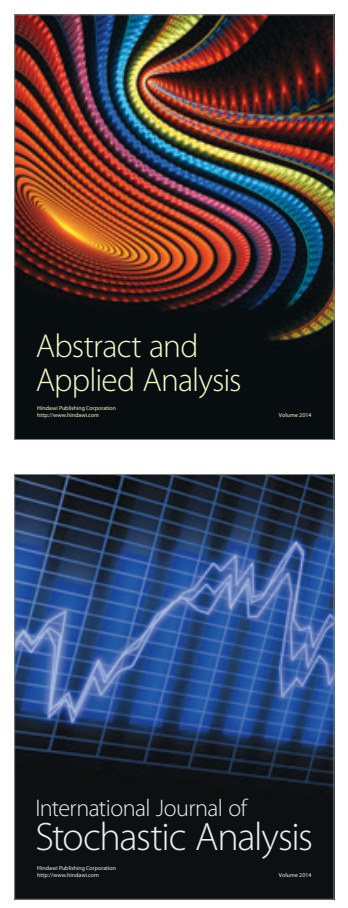

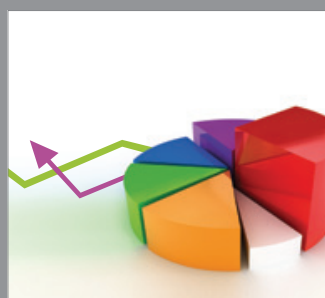

ournal of

Probability and Statistics

Promensencen
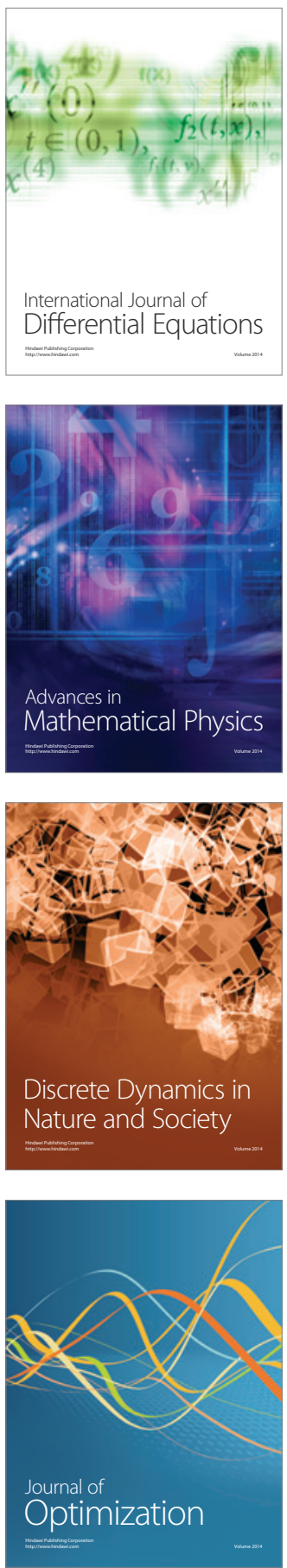